\title{
Anatomical variations of the superficial ulnar artery: case series observed on historical specimens prepared by Ludwik Karol Teichmann
}

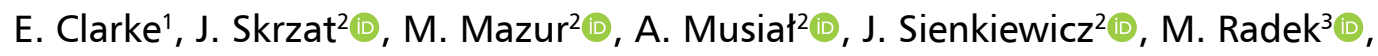 \\ M. Polguj'10, G. Wysiadecki' (1) \\ ${ }^{1}$ Department of Normal and Clinical Anatomy, Chair of Anatomy and Histology, Medical University of Lodz, Poland \\ ${ }^{2}$ Department of Anatomy, Jagiellonian University Medical College, Krakow, Poland \\ ${ }^{3}$ Department of Neurosurgery, Spine and Peripheral Nerve Surgery, Medical University of Lodz, University Hospital \\ WAM-CSW, Lodz, Poland
}

[Received: 13 September 2020; Accepted: 15 November 2020; Early publication date: 9 February 2021]

Background: This report presents a series of cases representing variant origin and course of the ulnar artery, namely the superficial ulnar artery (SUA), observed during the review of collection of historical specimens prepared in the $19^{\text {th }}$ century by the prominent Polish anatomist Ludwik Karol Teichmann and his collaborators, exhibited in the Anatomy Museum of Jagiellonian University Medical College. Results: Three distinct variants of the SUA were found on anatomical specimens of the upper limb with arteries injected by using Teichmann's method. In case no. 1, the SUA originated from the brachial artery slightly above the interepicondylar line of the humerus. This aberrant vessel gave off the common interosseous artery in the upper half of the cubital fossa and then ran superficially to the bicipital aponeurosis and over the muscles arising from the medial epicondyle of the humerus. The cases no. 2 and 3 involved two similar variants of the superficial artery in which the common interosseous artery arose from the radial artery. In the unique case no. 4, the SUA arose from the radial artery.

Conclusions: The SUA shows diverse anatomy regarding its topography and blood supply territory. Orthopaedic, hand, and plastic surgeons should be aware of anatomic variations of the SUA both in planning and in conducting surgeries of the upper limb. (Folia Morphol 2022; 81, 1: 227-233)

Key words: anatomic variation, arteries, brachial artery, common interosseous artery, radial artery, superficial ulnar artery, ulnar artery, superficial brachioulnoradial artery

\section{INTRODUCTION}

In the upper limb, the brachial artery is typically divided into two terminal branches; the radial and ulnar artery, at the level of the cubital fossa and slightly below the interepicondylar line of the humerus (Fig. 1) $[16,28]$. The radial artery courses under the cover of the brachioradialis muscle, turns back at the wrist below some slender tendons that go to the thumb and passes through the 1st metacarpal space to the palmar surface of the hand to form the deep palmar arch [16, 19-22, 28]. The ulnar artery gives off the common interosseous artery and passes deep to the

Address for correspondence: G. Wysiadecki, PhD, Department of Normal and Clinical Anatomy, Chair of Anatomy and Histology, Medical University of Lodz, ul. Żeligowskiego 7/9, 90-752 Łódź, Poland, e-mail: grzegorz.wysiadecki@umed.lodz.pl

This article is available in open access under Creative Common Attribution-Non-Commercial-No Derivatives 4.0 International (CC BY-NC-ND 4.0) license, allowing to download articles and share them with others as long as they credit the authors and the publisher, but without permission to change them in any way or use them commercially. 


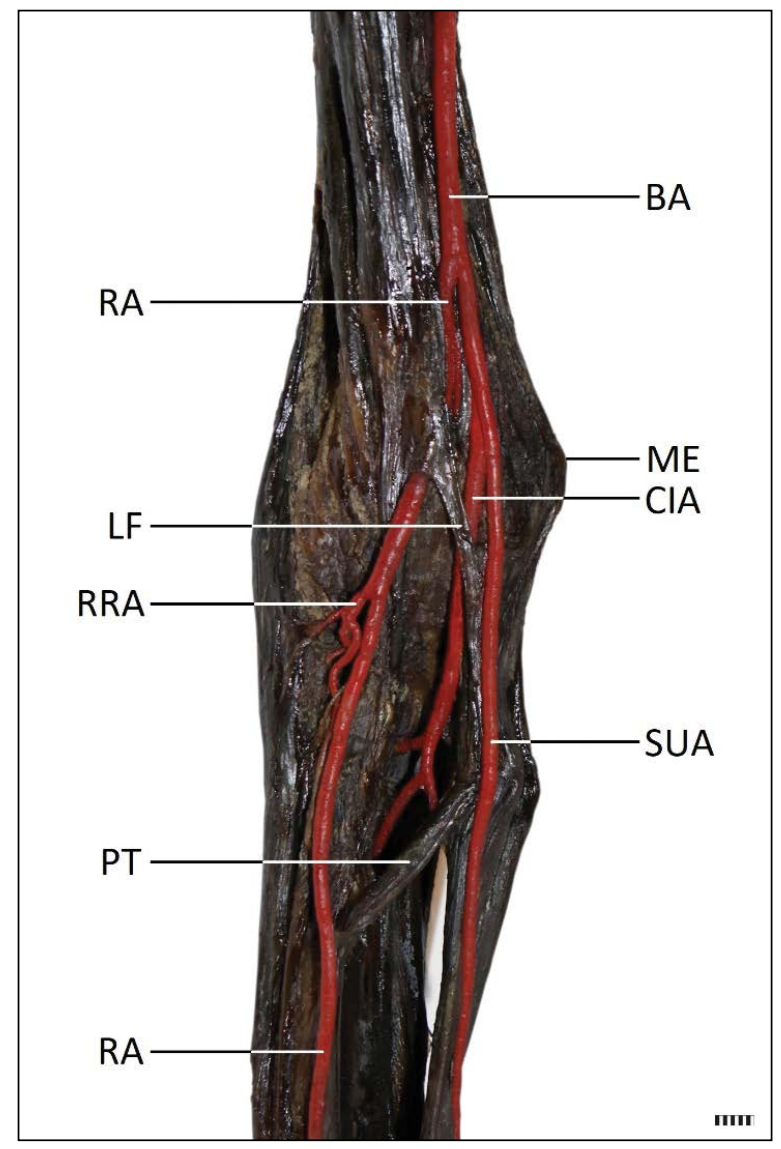

Figure 1. Variant of the superficial ulnar artery (SUA). Specimen described as case no. 1. Anterior view to the specimen of the right upper limb. The SUA originates from the brachial artery slightly above the interepicondylar line of the humerus. This aberrant vessel gives common interosseous artery in the upper half of the cubital fossa and then ran superficially to the bicipital aponeurosis (lacertus fibrosus) and over the muscles arising from the medial epicondyle of the humerus. In the lower third of the forearm, aberrant (superficial) ulnar artery followed its typical course. On this specimen the radial artery shows typical morphology. Scale bar shows $10 \mathrm{~mm}$; $\mathrm{BA}$ - brachial artery; $\mathrm{CIA}$ - common interosseous artery; LF — lacertus fibrosus; ME — medial epicondyle; PT — pronator teres muscle; RA — radial artery; RRA — radial recurrent artery.

ulnar head of the pronator teres muscle as well as under the flexor digitorum superficialis muscle. At the wrist, the ulnar artery runs superficially to the flexor retinaculum and terminates as the superficial palmar arch on the palmar surface of the hand [16, 19-22, 28]. Diverse variations of this arrangement may be observed; however, most of the anomalies involve the radial artery $[3,10-12,14,19-22,30,32]$. The superficial ulnar artery (SUA) is an anatomical variation in which the ulnar artery courses superficial to the flexors of the forearm. Such atypical vessel may arise directly from axillary, brachial or superficial brachial artery $[1-9,13,14,17-26,31-33]$. The incidence of this anatomical variation in adults ranges between $0.67 \%$ and $7 \%[14,20-23]$.

Research on the anatomical variations of blood vessels flourished between the $18^{\text {th }}$ and $20^{\text {th }}$ centuries $[9,14,19-22,27,29]$. In this report, we described a series of cases representing various origin and course of the SUA. All those anatomical variations were observed during the review of collection of historical specimens prepared in $19^{\text {th }}$ century by prominent Polish anatomist Ludwik Karol Teichmann [15] and his collaborators, exhibited in the Anatomy Museum of Jagiellonian University Medical College. Novelty of the study was in-depth anatomical description and a comparison of three different variants of the SUA located exclusively in the cubital fossa and forearm. The course, topography and branching pattern of those aberrant vessels were traced. The issue of classification and anatomical terminology was also discussed in the context of observed cases. Thus, our report may supplement anatomical description of the SUA existing in anatomical literature. It is especially true regarding the origin of the common interosseous artery, ulnar recurrent branches and formation of the superficial palmar arch in presence of this aberrant artery. As the anatomical variations of the arterial system in upper limbs may be a challenging clinical issue, this report may help to better understand in detail the diverse anatomy of the SUA, including its topography and blood supply territory.

\section{CASE REPORT}

Three distinct anatomical variants of the SUA were found on selected historical dry anatomical specimens of the upper limb, being a part of the collection presented in the Anatomy Museum (originally Theatrum Anatomicum) of Jagiellonian University Medical College. All those specimens varied regarding branching pattern and distribution of the main arterial trunks. Teichman introduced advances in techniques of the corrosion casting by developing mass for cold injections [15]. Using cold mass to prepare anatomical specimens did not damage the tissues. Teichman's mass applied to inject blood vessels was obtained using glass putty. The obtained images of the blood vessels were a very accurate reflection of true anatomical relationships. The diameters of radial and SUAs were measured at the level of the wrist to facilitate comparison of contribution of those vessels in hand blood supply. The measurements of external diameter 


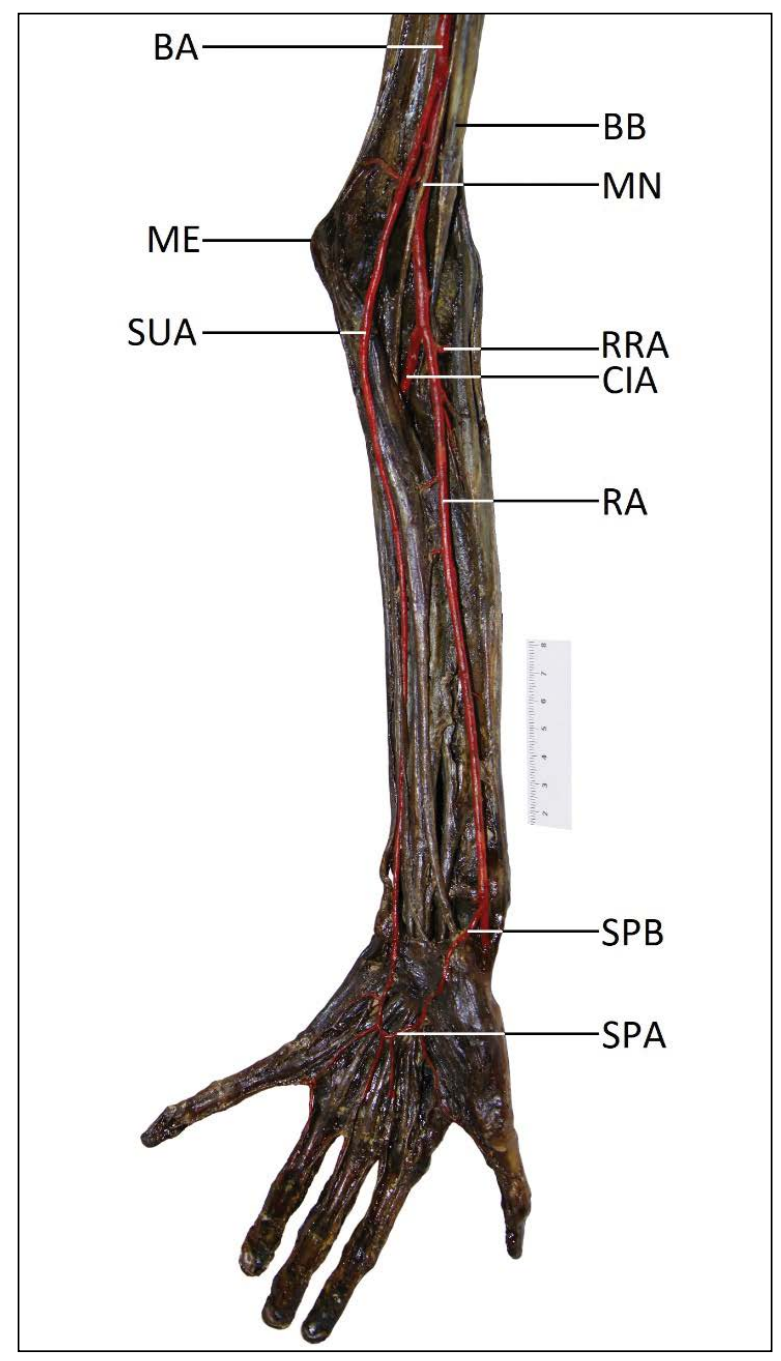

Figure 2. Variant of the superficial artery in which the common interosseous artery arise from the radial artery. Specimen described as case no. 2. Anterior view to the specimen of the left upper limb. In this case, the superficial ulnar artery (SUA) runs superficially to the flexors of the forearm and obtains position typical for the ulnar artery in the lower third of the forearm; BA - brachial artery; $\mathrm{BB}$ - biceps brachii muscle (tendon); $\mathrm{CIA}$ - common interosseous artery; ME — medial epicondyle; MN — median nerve; RA radial artery; RRA — radial recurrent artery; SPA — superficial palmar arch; SPB - superficial palmar branch of the radial artery.

of those arterial trunks were taken with a Digimatic Calliper (Mitutoyo Corporation, Kawasaki-shi, Kanagawa, Japan). Measurements were performed by two independent researchers. Each measurement was repeated twice and an average was calculated as final result, with an accuracy of two decimal places.

\section{Case 1}

In this case (specimen of the right upper limb), the SUA originated from the brachial artery above the cubital fossa, i.e. $51.5 \mathrm{~mm}$ proximally to the interepicondylar line of the humerus (Fig. 1). This aberrant vessel gave off common interosseous artery in the upper half of the cubital fossa, just at the level of the interepicondylar line. The common interosseous artery ran deep to the bicipital aponeurosis (Fig. 1) and gave off the ulnar recurrent arteries. The SUA ran superficially to the bicipital aponeurosis (lacertus fibrosus) and over the muscles arising from the medial epicondyle of the humerus (Fig. 1). No ulnar recurrent arteries were found on this specimen. In the lower third of the forearm $(141 \mathrm{~mm}$ below the medial epicondyle, with a forearm length of $276 \mathrm{~mm}$ ), aberrant (superficial) ulnar artery followed a typical course (Fig. 1). On this specimen the radial artery was typical. It ran under the bicipital aponeurosis, gave off radial recurrent artery and took the course along the lateral border of the brachioradialis muscle. The diameters of the SUA and the radial artery at the level of the wrist were $2.43 \mathrm{~mm}$ and $3.19 \mathrm{~mm}$, respectively. The hand was removed on this specimen, so the palmar arches couldn't be assessed.

\section{Case 2 and 3}

Both cases involved similar variants of the arterial pattern of the upper limb, in which the SUA was present, while the common interosseous artery arose from the radial artery (Fig. 2). In case no. 2 (specimen of the left upper limb), the SUA originated from brachial artery in the lower third of the arm, $65.5 \mathrm{~mm}$ above the interepicondylar line of the humerus (Fig. 2). In the case no. 3 (specimen of the left upper limb), the SUA originated from the brachial artery, slightly $(7.2 \mathrm{~mm})$ above the level of the interepicondylar line of the humerus. Thus, in both cases, the division of the brachial artery into the terminal branches (in this variant the terminal branches were radial and SUAs) was located in the distal arm, above the typical level. In both cases, the common interosseous artery branched off the radial artery (Fig. 2). It may be assumed that in this variant of the forearm arterial supply, the common interosseous artery replaced missing ulnar artery of typical location with respect to its muscular branches to the forearm flexors. On its course in the forearm, the SUA ran superficially to the flexors of the forearm and obtained position typical for the ulnar artery, i.e. between the flexor carpi ulnaris and flexor digitorum superficialis, in the lower third of the forearm (Fig. 2). In case no. 2, the typical 


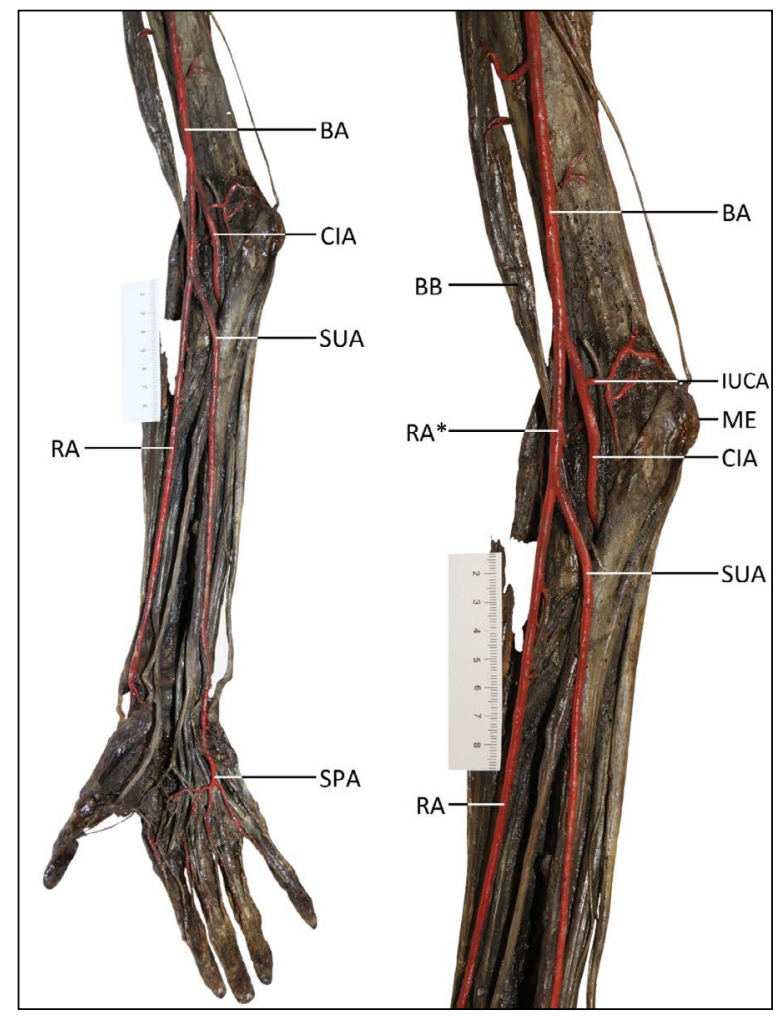

Figure 3. The superficial ulnar artery (SUA) arising from the radial artery (RA). Specimen described as case no. 4. Anterior view of the specimen of the right upper limb. The right side of this figure shows general view, the left side shows magnification of the anterior surface of the distal arm and proximal forearm. The brachial artery $(B A)$ is divided into the radial and common interosseous artery (CIA). The CIA resembles incomplete ulnar artery, it gives a single steam of the ulnar recurrent artery, and supplies flexors of the forearm, however, its territory does not involve the hand. In the lower half of the cubital fossa, the SUA branches off from the RA. This aberrant vessel crosses obliquely anterior group of the forearm muscles. At the level of wrist, it obtains a typical position for the ulnar artery; BB - biceps brachii muscle; IUCA — inferior ulnar collateral artery (anastomotica magna artery); $\mathrm{ME}$ — medial epicondyle; $\mathrm{RA}^{*}$ — initial part of radial artery (resembling superficial brachioulnoradial artery); SPA — superficial palmar arch.

position was obtained $164 \mathrm{~mm}$ below the medial epicondyle of the humerus (with forearm length of $267 \mathrm{~mm}$ ), while in the case no. 3 the normal position was obtained $182 \mathrm{~mm}$ below the medial epicondyle of the humerus (with the forearm length of $243 \mathrm{~mm}$ ). The diameters of the SUA and the radial artery at the level of the wrist were accordingly $2.11 \mathrm{~mm}$ and $3.15 \mathrm{~mm}$ in the case no. 2 , while the same diameters measured in the case no. 3 were $2.65 \mathrm{~mm}$ for the SUA and $2.95 \mathrm{~mm}$ for the radial artery. In both cases, the SUA contributed to blood supply of the hand; however, some differences in formation of the superficial palmar arch were found between those two cases.
In the case no. 2, the superficial palmar arch was of closed radio-ulnar type, in which contribution from the SUA formed an anastomosis with the superficial palmar branch of the radial artery (Fig. 2). In the case no. 3 , the superficial palmar arch was also radio-ulnar type (both radial and ulnar artery contributed to its formation). However, the arch was opened in this case with no anastomosis between the parts of the arch formed by the superficial palmar branch of the radial artery and SUA. In the case no. 3, the territory of the radial artery involved the princeps pollicis artery and the first metacarpal space, while the SUA has given off the common digital arteries to the second, third and fourth metacarpal spaces.

\section{Case 4}

In this rare case of the forearm arterial pattern observed on specimen of the right upper limb, the SUA arose from the radial artery. The brachial artery was divided $36.6 \mathrm{~mm}$ above interepicondylar line of the humerus into the radial artery and common interosseous artery (Fig. 3). The common interosseous artery gave off the inferior ulnar collateral artery and then supplied flexor muscles of the forearm. However, its blood supply territory did not involve the hand. The radial artery showed a typical course. However, in the lower half of the cubital fossa, $26 \mathrm{~mm}$ below the interepicondylar line of the humerus, the SUA branched off from the radial artery (Fig. 3). This aberrant vessel crossed obliquely anterior group of the forearm muscles. Only at the level of wrist, it obtained a position typical for the ulnar artery and joined the Guyon's canal (fibro-osseous tunnel extending from the transverse carpal ligament at the proximal aspect of the pisiform to the origin of the hypothenar muscles at the hook of hamate). The SUA in this case terminated in the incomplete superficial palmar arch (with no superficial palmar branch of the radial artery contributing to its formation). This variant may also be called the "ulnar type" of the superficial palmar arch. The princeps pollicis artery arose from the radial artery in this specimen. The diameters of the SUA and the radial artery at the level of the wrist were $2.91 \mathrm{~mm}$ and $3.34 \mathrm{~mm}$, respectively.

\section{DISCUSSION}

\section{Outline of embryology}

According to the classic "sprouting" theory, the arteries of the developing upper limb arise successively from a single trunk of the axial artery [27]. However, 


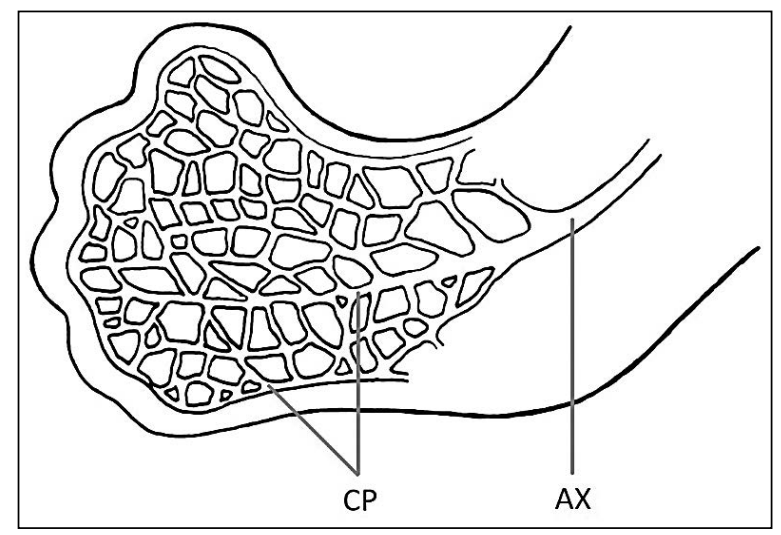

Figure 4. Capillary plexus (CP) in the developing upper limb; $\mathrm{AX}$ - axial artery differentiated within the arm region (precursor trunk for the brachial artery). This figure is a modification of the drawing taken from Wysiadecki et al. (2017) under the terms of the Creative Commons Attribution 4.0 International License (https:// creativecommons.org/licenses/by/4.0/), which permits unrestricted use, distribution, and reproduction in any medium.

based on recent theories, the precursor trunks for the main arteries of the upper limb develop from the primitive capillary plexus forming "vascular labyrinth" (Fig. 4). Based on this model it may be presumed that the dominant vascular channels in the developing upper limb gradually differentiate as a result of capillary remodelling. This remodelling may continue until the formation of the muscular membrane in the developing arteries [20-22]. Thus, an aberrant course of some arteries may result from plexiform appearance of the upper limb arteries during early stages of the ontogenesis. This model may also be useful to explain unusual variations of the definitive arterial pattern that occasionally occur during arterial development [20-22]. Examples of the results of such an atypical development of the ulnar artery have been presented in this report.

\section{Variants and classification of the SUA}

The SUA may originate at a variable level: within the axillary fossa or in the arm or in the cubital fossa $[1-9,13,14,17-26,31-33]$. It may provide an accessory ulnar recurrent artery, radial recurrent artery, or interosseous artery. However, in some variants of the SUA the ulnar recurrent or/and the common interosseous arteries may arise from the radial artery. Thus, different arterial patterns involving the presence of the SUA were described in the medical literature [1-9, 13, 14, 17-26, 31-33]. It should be emphasized, however, that there are difficulties in classification of individual variants of the SUA. Our case no. 1 resem- bles a situation where the typical termination of the brachial artery is shifted upwards. The initial segment of the SUA gives off the common interosseous artery, which is similar to typical anatomical relationships.

Another pattern, i.e. bifurcation of the brachial artery into a common radial-interosseous trunk and SUA, was described by Narayanan and Murugan [18]. This variant resembles cases no. 2 and 3 described in our report. However, based on the classification provided by Bergman et al. [3], this pattern may also be described as the radial origin of the common interosseous artery. Such classification was applied in our report. A different view for classification of this pattern was presented by Shankar and Veeramani [26], who stated that when the superficial artery is present, the brachial artery commonly terminates as the radial and common interosseous artery. Another way in which Gruber [9] and Rodríguez-Niedenführ et al. [21] describe the presence of the SUA in the forearm is partial duplication of the ulnar artery. According to those authors, partial duplications of the ulnar artery involves the presence of two vessels - the superficial ulnar artery reaching the palm and the rudimentary "normal" ulnar artery, sending of the ulnar recurrent artery, the common interosseous arteries and terminating as small muscular branches to the forearm flexor muscles. However, as Rodríguez-Niedenführ et al. [21] stress, many authors who reported the SUA, have observed the origin of the ulnar recurrent artery from the interosseous arteries, but they did not consider such variant as a duplication.

Based on the review of existing literature, we did not find a case of a radial origin of the SUA. Only the radial origin of the median artery was reported [3]. However, in the light of recent classifications [20-22], our case no. 4 may resemble the superficial brachioulnoradial artery. Rodríguez-Niedenführ et al. [21] define the superficial brachioulnoradial artery as "a superficial brachial artery branching at the elbow level into the radial and ulnar arteries coursing over the superficial forearm flexors and coexisting in the whole arterial pattern of the limb with a normal brachial artery that continues as the common interosseous trunk". The incidence of this variant ranges from $0.13 \%$ to $1.47 \%[17,21]$. However, the superficial brachioulnoradial artery most often arises from the proximal half of the brachial artery and bifurcates proximal to the interepicondylar line of the humerus [1, 2, 20-22]. In our case, the common trunk for the radial and SUA took origin in the distal quarter 
of the forearm ( $36 \mathrm{~mm}$ above the interepicondylar line of the humerus) and undergo division below the interepicondylar line of the humerus. Thus, double classification may be applied regarding this case.

The SUA, when present, terminates by joining the normal position in distal part of the forearm and hand [1-9, 13, 14, 17-26, 31-33]. In most cases, however, the radial artery seems to be the dominant vessel in the distal forearm and, consequently, constitutes the major source of vascularisation to the hand $[12,14$, $16,20-22]$. In all our cases the SUA was less dominant at the level of the wrist. The SUA may also be accompanied by anomalies of the palmar arches or forearm muscles [31-33].

\section{Clinical significance}

The SUA which runs beneath the skin may become vulnerable to iatrogenic or self-inflicted injuries $[1,4-8,14,17,23-25,31]$. Presence of the SUA may influence vascular and reconstructive procedures as well as evaluation of angiographic examination $[1,4-8,14,17,23-25,31]$. An unexpected atypical artery, running superficially, may also be a cause of complications during medical procedures, because incidental damage of such a vessel may cause distal limb ischaemia [14, 23].

Reports regarding the presence of SUA in a variety of clinical situations occasionally occur in medical literature. For example, Salunke et al. [23] reported a case of 22-year-old male who had sustained a glass cut injury to his right forearm and bleeding from the damaged radial artery. During revision of the wound, a large but intact superficial artery was found on the ulnar side of the surgical site. The diagnosis of SUA was confirmed in this case based on ultrasound Doppler examination and clinical assessment of the absence of the ulnar artery in its normal position [23]. Chin and Singh [5] conclude, based on their clinical experience, that SUA may cause a potential hazard in patients with difficult venous access. Those authors stress close relations of the SUA to the basilic vein which cause, that this aberrant artery may be at risk of unintended vascular puncture. They describe a case of a 53-year-old former heroin addict (which caused venous access difficulties), to gain intravenous access, a "non-pulsatile superficial vessel" was found in the right ventro-medial forearm, that resembled a venous channel. However, during an attempt at cannulation, blood was observed to flow rapidly up into the intravenous fluid tubing [5]. The cannulated vessel was identified as SUA. Chin and Singh [5] stress, that presence of the aberrant SUA should be kept in mind when the venous access is performed in the antecubital fossa or on the ventro-medial aspect of the forearm. Schonauer et al. [24], in turn, stress that SUA is a rare finding but shows significant surgical implications. When properly diagnosed, the presence of the SUA may also help during reconstructive surgeries; the SUA flap may be an excellent solution for soft tissue reconstruction [7, 24].

Salunke et al. [23] proposed a series of clinical recommendations to reduce the risk of complications when dealing with unexpected vascular variations. Those recommendations involve: using of intraoperative Doppler ultrasound during reconstructive and orthopaedic procedures, tracing arterial vessels at their normal anatomical location while using neurovascular structures as landmarks and careful preoperative assessment of the vascular anatomy.

\section{Limitations of the study}

There are some limitations as a result of the time and method of the specimen creation. For instance, not all branches of the common interosseous artery could be traced because it was impossible to reach the deeper muscle layers without damaging of the specimens. However, so far researchers refer to historical sources that still may be an inspiration for in-depth research on anatomical variations $[3,9,14,19,21$, 22]. Finally, the analysis of anatomical variations can contribute to obtaining an actual, not idealised image of the inside of the human body, which is crucial in everyday clinical practice [34].

\section{CONCLUSIONS}

The SUA shows diverse anatomy regarding its topography and blood supply territory. It may originate from the brachial artery in the lower third of the arm. It may give off the common interosseous artery, however in some instances the common interosseous may arise from the radial artery. The ulnar recurrent branches may also be absent. In rare cases the superficial ulnar may arise below the interepicondylar line of the humerus, from the radial artery. The blood supply territory of the SUA may thus be highly variable.

\section{Acknowledgements}

This report was prepared to commemorate Ludwik Karol Teichmann, one of the greatest Polish and world anatomists, who performed excellent research on the 
lymphatic system, described haemin crystals ("Teichmann's crystals") and introduced numerous advances in techniques of the anatomical preparations.

\section{Conflict of interest: None declared}

\section{REFERENCES}

1. Ariyo $\mathrm{O}$, Fenderson B. A variant of the classical superficial brachioulnoradial artery: morphology and clinical significances. Surg Radiol Anat. 2016; 38(6): 751-753, doi: 10.1007/ s00276-015-1605-6, indexed in Pubmed: 26831325.

2. Atlasi MA. A brachioulnoradial artery: a short report. Surg Radiol Anat. 2014; 36(1): 99-101, doi: 10.1007/s00276013-1126-0, indexed in Pubmed: 23645170.

3. Bergman RA, Afifi AK, Miyauchi R. Illustrated Encyclopedia of Human Anatomic Variation: Opus II: Cardiovascular System: Arteries: Upper Limb. 2015. https://www.anatomyatlases.org/AnatomicVariants/Cardiovascular/Text/ Arteries/Ulnar.shtml.

4. Casal $D$, Pais $D$, Toscano $T$, et al. A rare variant of the ulnar artery with important clinical implications: a case report. BMC Res Notes. 2012; 5: 660, doi: 10.1186/1756-05005-660, indexed in Pubmed: 23194303.

5. Chin KJ, Singh K. The superficial ulnar artery: a potential hazard in patients with difficult venous access. Br J Anaesth. 2005; 94(5): 692-693, doi: 10.1093/bja/aei548, indexed in Pubmed: 15814810.

6. D'Costa S, Shenoy BM, Narayana K. The incidence of a superficial arterial pattern in the human upper extremities. Folia Morphol. 2004; 63(4): 459-463, indexed in Pubmed: 15712144.

7. Devansh A. Superficial ulnar artery flap. Plast Reconstr Surg. 1996; 97(2): 420-426, doi: 10.1097/00006534199602000-00022, indexed in Pubmed: 8559826.

8. Fadel RA, Amonoo-Kuofi HS. The superficial ulnar artery: development and surgical significance. Clin Anat. 1996; 9(2): 128-132, doi: 10.1002/(SICl)10982353(1996)9:2<128::AID-CA5>3.0.CO;2-D, indexed in Pubmed: 8720787.

9. Gruber W. Über die Arteria mediana antibrachii superficialis, Arteria ulnaris antibrachii superficialis und Duplicität der Arteria ulnaris. Arch Anat Physiol Wisen Med. 1867: 668-687.

10. Haładaj R, Wysiadecki G, Dudkiewicz Z, et al. The high origin of the radial artery (brachioradial artery): its anatomical variations, clinical significance, and contribution to the blood supply of the hand. Biomed Res Int. 2018; 2018: 1520929, doi: $10.1155 / 2018 / 1520929$, indexed in Pubmed: 29992133.

11. Haładaj R, Wysiadecki G, Polguj M, et al. Hypoplastic superficial brachioradial artery coexisting with atypical formation of the median and musculocutaneous nerves: a rare combination of unusual topographical relationships. Surg Radiol Anat. 2019; 41(4): 441-446, doi: 10.1007/ s00276-019-02183-1, indexed in Pubmed: 30652211.

12. Haerle $M$, Häfner HM, Dietz $K$, et al. Vascular dominance in the forearm. Plast Reconstr Surg. 2003; 111(6): 1891-1898, doi: 10.1097/01.PRS.0000057529.76413.D7, indexed in Pubmed: 12711949.

13. Mannan A, Sarikcioglu L, Ghani S, et al. Superficial ulnar artery terminating in a normal ulnar artery. Clin Anat. 2005; 18(8): 602-605, doi: 10.1002/ca.20150, indexed in Pubmed: 16187323.

14. McCormack L, Cauldwell EW, Anson BJ. Brachial and antebrachial arterial patterns; a study of 750 extremities. Surg Gynecol Obstet. 1953; 96(1): 43-54, indexed in Pubmed: 13015348

15. Musiał A, Zarzecki M, Gryglewski R, et al. Ludwik Karol Teichmann (1823-1895). Folia Med Cracov. 2017; 57(4): 41-54, doi: $10.24425 / 118114$
16. Nasr AY. The radial artery and its variations: anatomical study and clinical implications. Folia Morphol. 2012; 71(4): 252-262, indexed in Pubmed: 23197145.

17. Natsis K, Papadopoulou AL, Paraskevas G, et al. High origin of a superficial ulnar artery arising from the axillary artery: anatomy, embryology, clinical significance and a review of the literature. Folia Morphol. 2006; 65(4): 400-405, indexed in Pubmed: 17171623.

18. Narayanan S, Murugan S. Bifurcation of brachial artery into a common radial-interosseous trunk and superficial ulnar artery: a rare variation. Anat Sci Int. 2018; 93(3): 400-403, doi: 10.1007/s12565-017-0427-5, indexed in Pubmed: 29277855.

19. Quain R. Anatomy of the arteries of the human body. Taylor and Walton, London 1844: 326-337.

20. Rodríguez-Niedenführ M, Burton GJ, Deu J, et al. Development of the arterial pattern in the upper limb of staged human embryos: normal development and anatomic variations. J Anat. 2001; 199(Pt 4): 407-417, doi: 10.1046/j.14697580.2001.19940407.x, indexed in Pubmed: 11693301.

21. Rodríguez-Niedenführ M, Vázquez T, Nearn L, et al. Variations of the arterial pattern in the upper limb revisited: a morphological and statistical study, with a review of the literature. J Anat. 2001; 199(Pt 5): 547-566, doi: 10.1046/j.14697580.2001.19950547.x, indexed in Pubmed: 11760886.

22. Rodríguez-Niedenführ M, Vázquez T, Parkin I, et al. Arterial patterns of the human upper limb: update of anatomical variations and embryological development. Eur J Anat. 2003; 7(S1): 21-28.

23. Salunke AA, Nambi GI, Dhanwate AD, et al. Superficial ulnar artery: Clinical recommendations to avoid iatrogenic complications due to variation in arterial system. Niger Med J. 2014; 55(3): 276-277, doi: 10.4103/03001652.132071, indexed in Pubmed: 25013265.

24. Schonauer F, Marlino S, Turrà F, et al. Superficial ulnar artery perforator flap. J Craniofac Surg. 2014; 25(5): 1870-1871, doi: 10.1097/SCS.0000000000001061, indexed in Pubmed: 25102397.

25. Senanayake KJ, Salgado S, Rathnayake MJ, et al. A rare variant of the superficial ulnar artery, and its clinical implications: a case report. J Med Case Rep. 2007; 1: 128, doi: 10.1186/1752-1947-1-128, indexed in Pubmed: 17988391.

26. Shankar N, Veeramani R. Bilateral superficial ulnar arteries with an unusual arterial arch in the forearm. Int J Anat Var (IJAV. 2009; 2: 24-26.

27. Singer E. Embryological pattern persisting in the arteries of the arm. Anat Rec. 1933; 55(4): 403-409, doi: 10.1002/ ar.1090550407.

28. Standring S. Gray's Anatomy: The Anatomical Basis of Clinical Practice. Churchill Livingstone, Edinburgh, London 2016.

29. Von Ha. Anatomical description of the arteries of the human body. Thomas B Wait, Boston, MA 1813.

30. Wysiadecki G, Polguj M, Haładaj R, et al. Low origin of the radial artery: a case study including a review of literature and proposal of an embryological explanation. Anat Sci Int. 2017; 92(2): 293-298, doi: 10.1007/s12565-016-0371-9, indexed in Pubmed: 27631096.

31. Yadav PS, Ahmad QG, Shankhdhar VK, et al. Absence of the palmaris longus is a warning sign for avoiding the superficial ulnar artery 'trap'. Indian J Plast Surg. 2013; 46(1): 149-150, doi: 10.4103/0970-0358.113738, indexed in Pubmed: 23960326.

32. Yalcin B, Kocabiyik N, Yazar F, et al. Arterial variations of the upper extremities. Anat Sci Int. 2006; 81(1): 62-64, doi: 10.1111/j.1447-073X.2006.00110.x, indexed in Pubmed: 16526599.

33. Yazar F, Kirici $Y$, Ozan $\mathrm{H}$, et al. An unusual variation of the superficial ulnar artery. Surg Radiol Anat. 1999; 21(2): 155-157, doi: 10.1007/s00276-999-0155-1, indexed in Pubmed: 10399219.

34. Żytkowski A, Tubbs R, Iwanaga J, et al. Anatomical normality and variability: Historical perspective and methodological considerations. Transl Res Anat. 2021; 23: 100105, doi: 10.1016/j.tria.2020.100105. 